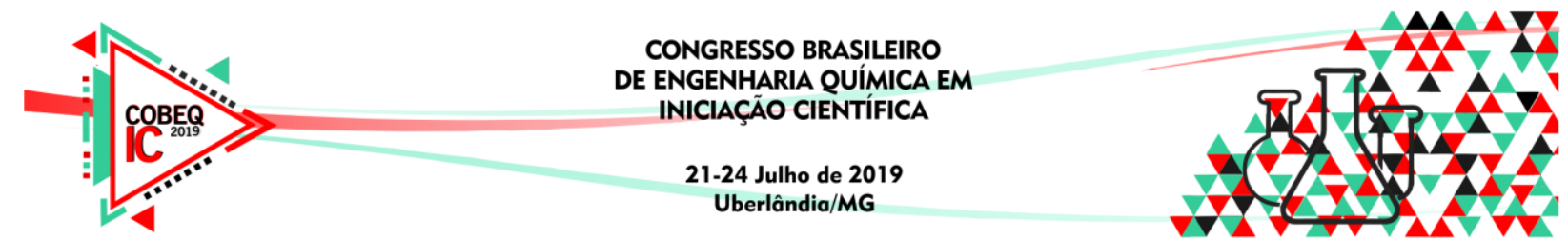

\title{
ESTUDOS INICIAIS DO DESENVOLVIMENTO DE UM SENSOR ELETROQUÍMICO MODIFICADO COM POLÍMEROS MOLECULARMENTE IMPRESSOS PARA DETECÇÃO DE ÍONS CHUMBO II EM AMOSTRAS REAIS
}

\author{
E. S. MARINHO ${ }^{1}$, L. M. F. LOPES $^{1}$, T. A.R. CORDEIRO ${ }^{1}$, L. F. FERREIRA ${ }^{1 *}$ \\ ${ }^{1}$ Universidade Federal dos Vales do Jequitinhonha e Mucuri, Instituto de Ciência e \\ Tecnologia \\ E-mail para contato: lucas.franco@ict.ufvjm.edu.br
}

\begin{abstract}
RESUMO - O chumbo é um metal pesado altamente tóxico para os seres humanos, que pode ser proveniente de diversas fontes como combustíveis, tintas, entre outras. Este trabalho buscou produzir um sensor à base de polímero molecularmente impresso (MIP), aplicado com especificidade para a molécula de chumbo, capaz de detectar íons de chumbo II em soluções de amostras reais. Para tal, utilizou-se eletrodos de carbono grafite e, através da técnica de voltametria cíclica foi realizada a eletropolimerização do monômero ácido 4 hidroxifenilacético (4-HFA) e nitrato de chumbo em solução de ácido perclórico

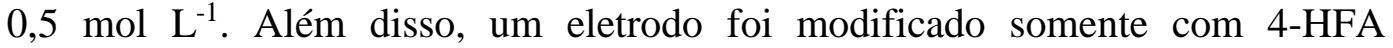
(polímero não impresso - NIP). A detecção de $\mathrm{Pb}^{2+}$ foi realizada através da técnica de voltametria de pulso diferencial e assim o comportamento de resposta dos eletrodos foi investigado. O eletrodo modificado com MIP mostrou uma resposta consideravelmente maior em comparação ao eletrodo com polímero não impresso (NIP). Isto indicou que os locais de reconhecimento de $\mathrm{Pb}^{2+}$ foram criados na estrutura no estágio de polimerização, comprovando a eficiência da plataforma desenvolvida. Como perspectiva do trabalho, estudos de otimização da plataforma e tendo como objetivo testes em amostras reais deverão ser realizadas.
\end{abstract}

\section{INTRODUÇÃO}

Os metais pesados são elementos considerados de baixa densidade que proporcionam efeitos danosos a saúde humana e ao meio ambiente, quando são expostos por longos períodos. São metais tóxicos, pois não são biodegradáveis e além disso se acumulam ao longo da cadeia alimentar (GUMPU, et al., 2015). Os metais mais comuns, encontrados principalmente em águas contaminadas, são: arsênio, cádmio, cromo, cobre, chumbo, níquel e zinco (JAISHANKAR, et al., 2014). Alguns metais pesados, como por exemplo o ferro, são necessários para o bom funcionamento do organismo dos seres humanos, porém em baixas concentrações. Elevadas taxas desses metais podem afetar o funcionamento das células, sendo prejudiciais à saúde. Metais, como o chumbo, cádmio ou arsênio, até mesmo em baixas concentrações são danosos ao organismo (GUMPU, et al., 2015).

O chumbo é altamente tóxico, e está presente principalmente em combustíveis (como a gasolina), tintas, encanamentos, entre outras fontes (JAISHANKAR, et al., 2014). Contudo 


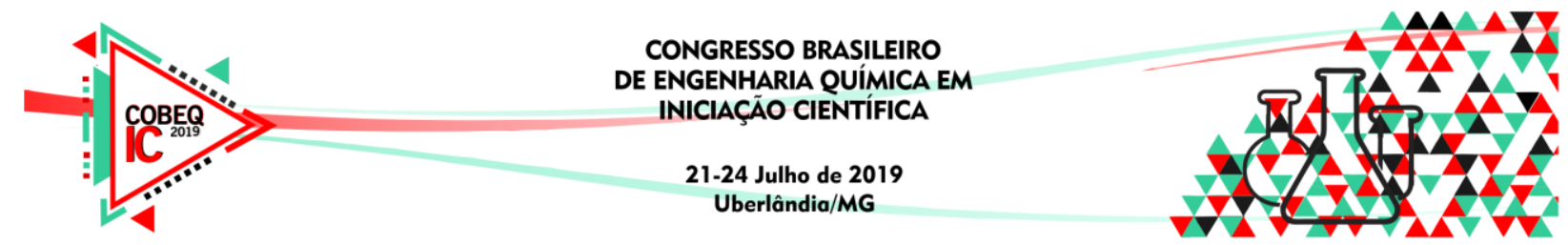

ele não desenvolve nenhuma função biológica, e sua exposição pode levar a doenças neurodegenerativas (GUMPU, et al., 2015).

As técnicas mais comuns utilizadas para detecção de íons de metais são: emissão atômica de plasma acoplado induzido (ICP-AES), espectrometria de massa com plasma indutivamente acoplado (ICP-MS), espectrometria de absorção atômica com chama (FAAS), espectrometria de absorção atômica com forno de grafite (GFAAS) e espectrometria de emissão óptica com plasma indutivamente acoplado (ICP-OES). Essas técnicas tem a vantagem de serem rápidas e possuírem baixos limites de detecção, porém há dificuldade quando a amostra possui interferentes, além de terem um alto custo associado e precisarem de processos de pré-concentração da amostra antes de poderem ser analisadas (LI, et al., 2015).

Uma alternativa para detecção de amostras em baixas concentrações são os polímeros molecularmente impressos, também conhecidos como MIP's. Esses polímeros biomimetizam interações biológicas, como enzima substrato, ou seja, são altamente seletivos. Além disso apresentam alta estabilidade química e podem ser produzidos em larga escala (HASSAN, et al., 2018).

Os MIP's podem desempenhar o papel de sensores, uma vez que são sensíveis ao template utilizado na polimerização (UDOMSAP, et al., 2018). Os MIP's podem ser sintetizados por voltametria cíclica, uma técnica simples e de baixo custo, que facilita a reprodutibilidade do método. Essa técnica pode ser utilizada para a etapa de polimerização e formação do polímero impresso. Assim um monômero funcional e uma molécula molde (template) sofrerão co-polimerização, estes irão interagir de modo que a molécula molde estará ligada à estrutura do polímero, formando ligações cruzadas. Ao remover a molécula molde, o espaço determinado a mesma permanecerá na estrutura, assim como as interações. Esse fato é o que caracteriza a alta seletividade dos MIP's ao analito de interesse (HAUPT, et al., 1999).

Assim, considerando a alta toxicidade do chumbo à saúde humana e ao meio ambiente, este trabalho propôs o desenvolvimento sensor eletroquímico modificado com MIP visando estabelecer perspectivas para melhorias na detecção de tal metal. A metodologia utilizada é extremamente promissora, tendo como estudos posteriores a otimização e aplicação dessa plataforma em amostras reais.

\section{METODOLOGIA}

Os eletrodos de trabalho foram produzidos utilizando barras de grafite $(100 \times 0,6 \mathrm{~mm})$, provenientes da Alfa Aesar (99,9999\%) que foram cortadas em tamanhos menores e então colocados sobre bases de latão revestidas de Teflon®. Para fixar o grafite (espessura de aproximadamente $2 \mathrm{~mm}$ ) utilizou-se cola de prata, e Araldite ${ }^{\circledR}$ para garantir o isolamento do latão e da cola de prata. Logo em seguida esses eletrodos foram lixados em lixa d'água MIRKATM número 200, 1500 e 2000, para remoção de excessos de cola e garantir uma superfície plana. Para o polimento do eletrodo utilizou alumina 0,30 $\mu \mathrm{m}$ (Micropolish A, Buehler), onde depois desse procedimento o eletrodo foi limpo em água ultrapura, e seco usando gás nitrogênio. 


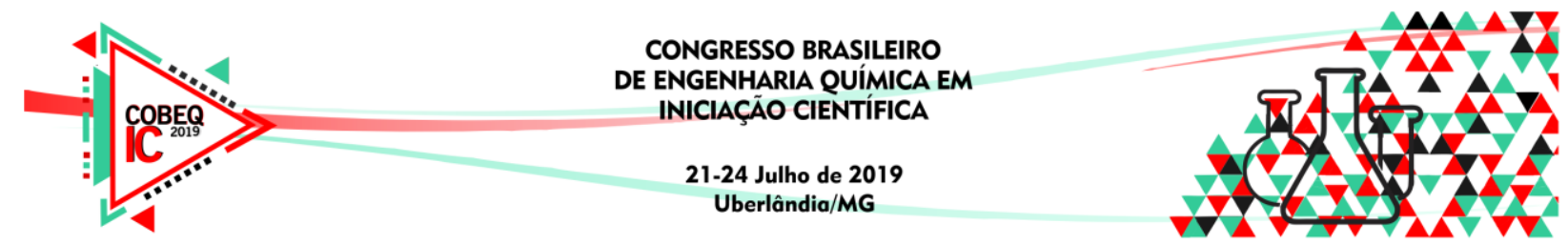

Antes de proceder a eletropolimerização, os eletrodos confeccionados foram submetidos a análises utilizando soluções de ácido sulfúrico $0,5 \mathrm{~mol} \mathrm{~L}^{-1}$ e ferrocianeto/ferricianeto de potássio $(5,00 \mathrm{mM}$ com $0,10 \mathrm{M}$ de $\mathrm{KCl})$ pelo método de voltametria cíclica para verificar sua eletroatividade.

A eletropolimerização do monômero 4HFA $2,5 \mathrm{mmollL}^{-1}$ foi realizada pela técnica de voltametria cíclica no qual foram realizados 100 ciclos de potencial com velocidade de varredura de $50 \mathrm{mV} / \mathrm{s}$, na faixa de potencial de $+0,00 \mathrm{a}+1,40 \mathrm{~V}$, na presença (MIP) e ausência (NIP) de nitrato de chumbo $\left(0,1 \mathrm{mmol} \mathrm{L}^{-1}\right)$ em solução de ácido perclórico $0,5 \mathrm{~mol} \mathrm{~L}^{-1}$. Após realizada a eletropolimerização os eletrodos foram analisados em solução de ácido clorídrico $\left(0,01 \mathrm{~mol} \mathrm{~L}^{-1}\right)$, com objetivo de verificar se houve uma alteração na resposta eletroquímica do eletrodo de grafite após o procedimento de eletropolimerização, utilizando a voltametria de pulso diferencial (VPD), com um pré-condicionamento de 30 segundos, na faixa de potencial de $-0,60$ a $-0,10 \mathrm{~V}$, com uma velocidade de varredura de $50 \mathrm{mV} / \mathrm{s}$.

Em seguida os eletrodos modificados foram colocados em um recipiente contendo uma solução de peróxido de hidrogênio $0,05 \mathrm{~mol} \mathrm{~L}^{-1}$ por 10 minutos para que a molécula molde seja retirada. Logo após, a resposta eletroquímica foi verificada e assim MIP e NIP foram expostos a uma solução de $\mathrm{Pb}\left(\mathrm{NO}_{3}\right)_{2} \quad 0,5 \mathrm{mmol} \mathrm{L}^{-1} \mathrm{em}$ diferentes tempos e analisados em VPD em solução de ácido clorídrico, para avaliar capacidade de ambas plataformas na detecção de chumbo.

\section{RESULTADOS E DISCUSSÃO}

Inicialmente, após o processo de eletropolimerização buscou-se comprovar a presença de chumbo no polímero formado através da técnica de VPD em solução de $\mathrm{HCl} 0,01 \mathrm{~mol} \mathrm{~L}^{-1}$, e assim verificar se o íon $\mathrm{Pb}^{2+}$ havia sido incorporado na estrutura do polímero após a eletropolimerização. Assim a Figura 1 (curva 1) representa a resposta do filme de 4-HFA formado na presença de $\mathrm{Pb}^{2+}$, em que é possível perceber a presença de $\mathrm{Pb}^{2+}$, uma vez que a resposta obtida apresentou um pico de corrente correspondente ao potencial de oxidação do mesmo, cerca de $-0,41 \mathrm{~V}$.

Figura 1 - Presença de $\mathrm{Pb}^{2+}$ no MIP e retirada de $\mathrm{Pb}^{2+}$.

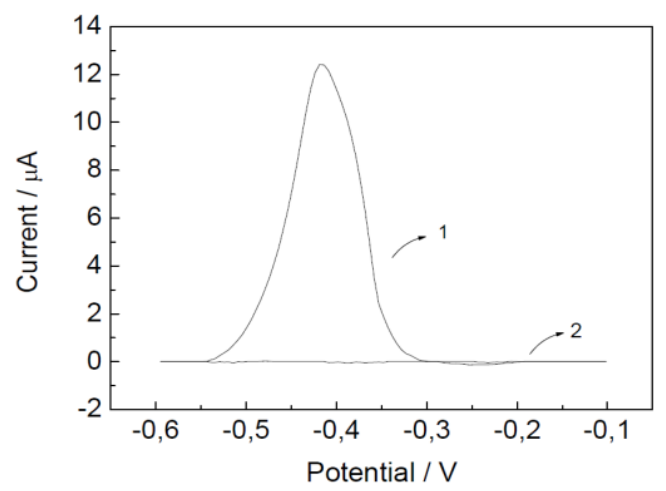

Para remoção do chumbo presente no polímero e consequente formação do MIP, a plataforma foi colocada em solução de $\mathrm{H}_{2} \mathrm{O}_{2}$ por 20 minutos. A Figura 1 (curva 2) representa 


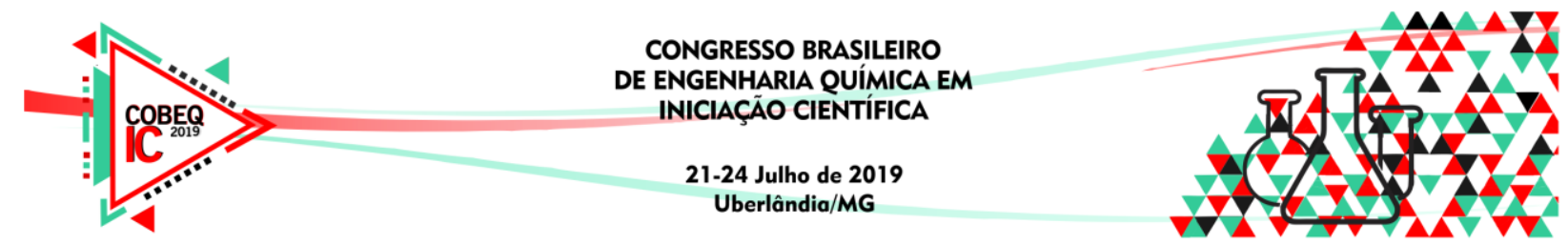

a resposta obtida para o eletrodo após esse tempo, onde pode-se perceber a ausência do pico obtido anteriormente, comprovando assim a eficiência na remoção do chumbo.

Após a remoção do chumbo, foram testadas a capacidade das plataformas MIP e NIP na determinação de $\mathrm{Pb}^{2+}$ foram testadas através da incubação do eletrodo em diferentes tempos e, posteriormente, análise eletroquímica. A Figura 2 mostra as respostas da VPD dos eletrodos NIP e MIP antes (curva 1) e após estarem em contato com a solução de $\mathrm{Pb}^{2+} 1 \times 10^{-4} \mathrm{~mol} \mathrm{~L}^{-1}$ por 10,20 e 30 minutos (curva 2,3 e 4 respectivamente).

Figura 2 - Curvas para NIP(A) e MIP (B) em 0, 10, 20 e 30 minutos.
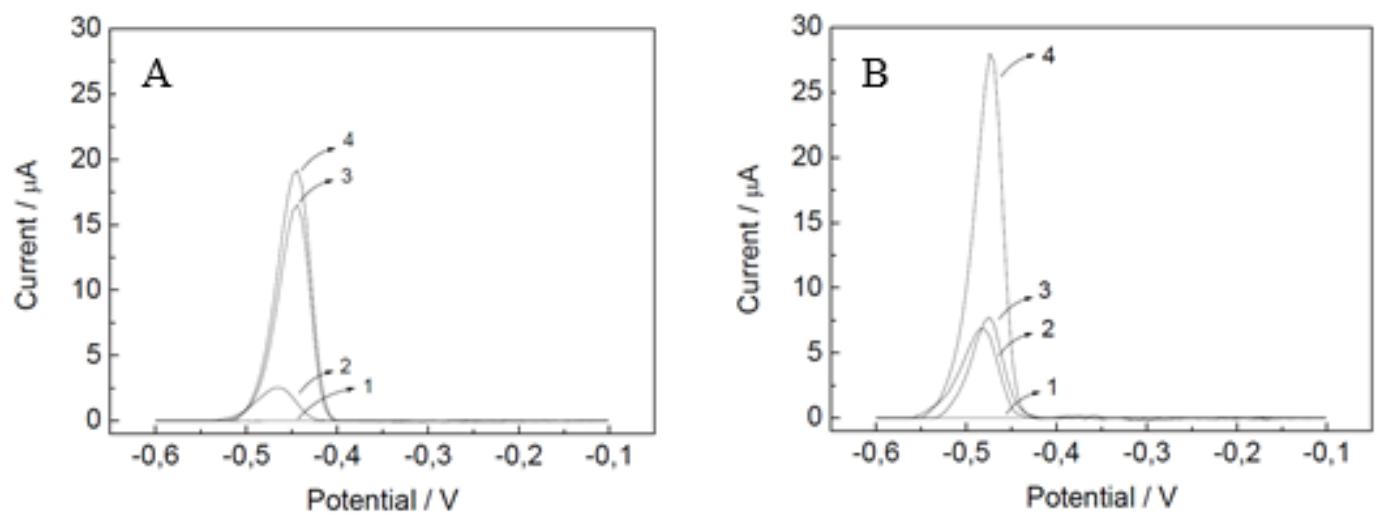

Observando a Figura 2, pode ser perceber que tanto MIP quanto NIP foram capazes de detectar a presença de $\mathrm{Pb}^{2+}$ na amostra, além disso é possível perceber a relação diretamente proporcional do tempo de incubação com o tamanho do pico, assim quanto maior o tempo de exposição do sensor à solução que contém chumbo, maior o pico apresentado.

Porém, pode-se perceber que o MIP apresenta maiores amplitudes dos picos apresentados em comparação com NIP em um mesmo intervalo de tempo. Isso possivelmente ocorre pois no MIP o $\mathrm{Pb}^{2+}$ está presente na estrutura do polímero, ligando neste através dos sítios ativos criados, enquanto no NIP esses íons se depositaram apenas em sua superfície. Mostrando assim uma melhora no sinal quando o MIP é utilizado para detecção de $\mathrm{Pb}^{2+}$. Além disso, avaliando o processo de detecção de chumbo para aplicações posteriores foi escolhido um tempo de incubação de 30 minutos para garantir a maior detecção de chumbo.

Assim, os resultados obtidos comprovam que o método foi eficaz para a produção de um sensor eletroquímico modificado com polímero molecularmente impresso que pode ser utilizado para detecção de $\mathrm{Pb}^{2+}$.

\section{CONCLUSÃO}

Os estudos iniciais realizados neste trabalho mostram resultados promissores para a produção de um sensor que seja capaz de detectar íons de chumbo II em amostras de concentração relativamente baixas. Mostrando ser uma plataforma viável pela sua praticidade e menor custo frente aos métodos já existentes de quantificação deste metal. 


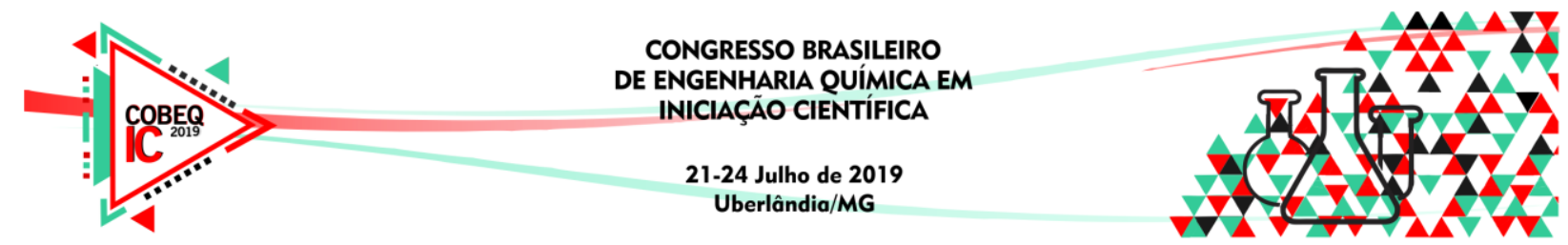

Para otimização da plataforma deverão ser realizados testes do efeito da composição do filme na resposta, ou seja, a melhor proporção 4-HFA/ $\mathrm{Pb}^{2+}$ a ser utilizada, testar a seletividade do sensor frente a adição de outros metais à plataforma, além da aplicação da plataforma em amostras reais.

\section{REFERÊNCIAS}

GUMPU, B. M.; SETHURAMAN, S.; KRISHNAN, M. U.; RAYAPPAN, B. B. J. A review on detection of heavy metal ion in water - An electrochemical approach. Sensors and Actuators B: Chemical, p. 515-533, 2015.

HASSAN, H. A. A.; MOURA, L. S.; ALI, H. M. F.; MOSELHY, A. W.; SOTOMAYOR, T. P. M.; PIVIDORI, I. M. Eletrochemical sensing of methyl parathion on magnetic molecularly imprinted polymer. Biosensors and Bioeletronics, v. 118, p. 181-187, 2018.

HAUPT, K.; MOSBACH, K. Molecularly Imprinted Polymers and Their Use in Biomimetic Sensors. Chem. Rev., v. 100, p. 2495-2504, 2000.

JAISHANKAR, M.; TSETEN, T.; ANBALAGAN, N.; MATHEW, B. B.; BEEREGOWDA, N. K. Toxicity, mechanism and health effects of some heavy metals. Interdisciplinary toxicology, v. 7, p. 60-72, 2014.

LI, Y.; HE, Q.; PENG, G. Dispersive liquid-liquid microextraction based on the solidification of floating organic drop followed by ICP-MS for the simultaneous determination of heavy metals in wastewaters. Spectrochimica Acta Part A: Molecular and Biomolecular Spectroscopy, v. 140, p. 156-161, 2015.

PILETSKY, A. S.; TURNER, P. F. A. Eletrochemical Sensors Based on Molecularly Imprinted Polymers. Electroanalysis, v. 14, n. 5, p. 317-323, 2001.

SANTOS, C. C. Desenvolvimento de plataformas eletroquímicas funcionalizadas com ácido poli(4-aminobenzóico) aplicadas em biossensores. Dissertação (Mestrado pelo Programa de Pós Graduação em Química) - Universidade Federal dos Vales do Jequitinhonha e Mucri, Diamantina, 2014.

SIMÕES, R. F. Desenvolvimento e caracterização de materiais de eletrodos modificados com polímeros condutores para a determinação eletroanalítica de pesticidas. Tese (Doutorado pela Área Interunidades em Ciência e Engenharia de Materiais) Universidade de São Paulo, São Carlos, 2005.

UDOMSAP, D.; BRISSET, H.; CULIOLI, G.; DOLLET, P.; LAATIKAINEN, K.; SIREN, H.; BRANGER, C. Eletrochemical molecularly imprinted polymer as material for pollutant detection. Materials TodayComunications, v. 17, p. 458-465, 2018. 